\title{
The additional impact of simulation based medical training to traditional medical training alone in advanced cardiac life support: a scenario based evaluation
}

\author{
ERKMAN SANRI ${ }^{1}$, SINAN KARACABEY ${ }^{1}$, SERKAN EMRE EROGLU ${ }^{2}, H A L D U N$ AKOGLU ${ }^{1}$, ARZU DENIZBASI $^{1}$ \\ 1 Department of Emergency Medicine, Marmara University Pendik Education and Research Hospital, Istanbul, Turkey \\ 2 Department of Emergency Medicine, Umraniye Training and Research Hospital, Istanbul, Turkey
}

Corresponding Author

Erkman Sanr

Department of Emergency Medicine

Marmara University Faculty of Medicine

Marmara Universitesi Pendik Egitim ve Arastirma Hastanesi

Acil Tip Anabilim Dali. Pendik / Istanbul, Turkey

Phone: +902166254545

Mobile: +905333164100

Fax: +902166254639

E-mail:erkmansanri@gmail.com

\section{ABSTRACT}

Objectives. The principal aim of medical education is to provide medical student with the fundamental knowledge and required skills that can be specifically used in real-life conditions such as high-quality cardiopulmonary resuscitation (CPR). Traditional medical training (TMT) is an effective method in Advanced cardiac life support (ACLS) training. Simulationbased medical training (SBMT), with the advancements in technology, is a relatively new, but a preferred ACLS training method since it implements a safe educational environment. We planned a scenario-based study to evaluate the additional impact of SBMT to TMT alone in ACLS training. Methods. This before-after type, comparative, cohort study was performed in a simulation center. One hundred thirtysix 6th grade medical students who took ACLS training with TMT on their emergency medicine clerkship were enrolled in 34 teams. All students managed a specific ACLS scenario before and after SBMT with a high-fidelity manikin. All data regarding chest compression, airway management, defibrillation and drug administration were recorded by the sensors of the highfidelity manikin.

Results. Median age was 23 and 51.5\% were male. After SBMT, we found significant increases in the successful CPR cycle rate and successful scenario completion rate (60.3\%; 61.8\%, respectively). Median time to chest compression (Tcc) and defibrilla- tion (Tdef) were significantly decreased after SBMT (1 sec., 1 sec., respectively). For the adequacy of chest compressions, compression depth, recoil, and frequency are all significantly increased after SBMT, $7.0 \mathrm{~mm}, 6.0 \mathrm{~mm}$ and $8.5 / \mathrm{min}$, respectively. Conclusion. SBMT in combination with TMT is a promising ACLS training method when compared to TMT alone.

Key words: simulation-based medical training, traditional medical training, high-fidelity manikin, CPR, ACLS

\section{INTRODUCTION}

The primary goal of medical training, especially in emergency medicine, is to provide medical student with the fundamental knowledge and required skills such as high-quality cardiopulmonary resuscitation (CPR), that can be used accurately in real life situations. (1) The administration of CPR following the American Heart Association (AHA) Guidelines has been shown to reduce mortality in patients suffering from cardiac arrest. $(2,3)$

In recent years, there has been a search for an education method that establishes the utilization of knowledge and skills in Advanced cardiac life support (ACLS) without causing adverse clinical outcomes. Traditional (didactic) medical training (TMT) is an efficient and well-experienced training method; on the other hand, with the advances in technology, the use of sim- ulation-based medical training (SBMT) is increasing since SBMT provides a safe and supportive educational setting, so that students can improve their performance without causing adverse clinical outcomes. (4-6)

Previous articles showed that SBMT for ACLS training is a more efficient method when compared to TMT alone. SBMT was studied not only on medical students but also on medical residents, nurses, respiratory therapists and dental students in ACLS training. (7-10) On the contrary, in $2002 \mathrm{Kim}$ et al. stated TMT is a more efficient method when compared to SBMT in ACLS training for medical students. (4) In 2012, a paper from Korea found that after one month of ACLS training, there was no significant difference between SBMT and TMT groups. (11)

In this study, we aim to evaluate the additional impact of SBMT by comparing the efficacy for the combination of SBMT and TMT, with TMT alone in ACLS training via a standard ACLS scenario.

\section{MATERIALS AND METHODS}

Study design, period and place

This before-after type, comparative, cohort study was conducted between January 2017 and December 2017 at a simulation center. Simulation center was 800 square meters, consisting of two high fidelity SimMan Essential, six low fidelity mani- 
kins, four simulation areas, closed circuit camera system, and brief-debrief room. All simulations were performed on a highfidelity manikin, which has an extensive list of features regarding BLS, ACLS and ATLS training (SimMan Essential, Laerdal Medical, Norway).

\section{Study population}

The study population consisted of undergraduate medical students at the 6th grade in Marmara University Faculty of Medicine. In this faculty, 6th-grade medical students observe and practice emergency medicine for two months during their emergency medicine clerkship rotation. Standard, didactic airway management, CPR, basic life support (BLS) and ACLS lessons were provided to each rotation group during their clerkship program as defined in the curriculum. Then, at the end of their clerkship, all students who agreed to participate to the study by signing informed consent forms were taken to the simulation center and randomly grouped in teams of 4 people. A one hour long briefing before the scenario was given in the briefing room. They managed a specific ACLS scenario as a team and their success parameters were recorded. Then, an interactive, practical, simulation-based medical ACLS training (SBMT) with the use of a high-fidelity manikin was performed. The same simulation case was repeated on the high-fidelity manikin. The changes in the success parameters after the SBMT were compared. A video-assisted debriefing was performed after all scenario evaluations had been completed. A total of 136 students were recruited and evaluated as 34 teams.

\section{Scenario}

The following ACLS scenario of an asystole case was selected. The simulated case was a 68-year- old female with a congestive heart failure (CHF) and chronic obstructive pulmonary disease (COPD). She was admitted to emergency department (ED) with the complaint of dyspnea lasting for 2 days. At the first contact, she had orthopnea and tachypnea with an arterial blood pressure of 200/120 mmHg. Her heart rate was $110 \mathrm{bpm}$, respiratory rate was 14/ min, and pulse $\mathrm{O} 2$ saturation was $88 \%$. On her physical examination, rales on both lung bases, and bilateral pretibial edema were recognized. Bedside ultrasonography (US) revealed a distended vena cava inferior with a diameter of $23 \mathrm{~mm}$. B-lines were present on the pulmonary US. Therefore, a case of decompensated CHF due to a hypertensive emergency was presented. According to the scenario, the patient's dyspnea worsened, continuous positive airway pressure (CPAP) was initiated, but she could not tolerate the mask and suddenly arrested. The simulation started at this point at the bedside of the patient.

\section{Data collection}

All data regarding the scenario were recorded by the sensors of the high-fidelity manikin for every action conducted, following the institutional Ethics Committee's approval (ID:09.2017.496). The data were sent to a central, dedicated server, where Laerdal Learning Application (LLEAP) was installed. Electronically collected data were as follows: time to chest compressions, the frequency of chest compressions, depth and recoil of chest compressions, the frequency of rescue breaths, time to defibrillation, defibrillation dose, drugs administered, and their doses. Demographics of the students and success at previously defined checkpoints of the scenario were evaluated by the educators and noted on a data sheet. Demographics of the students were then transferred to the same datasheet. Educators consisted of a simulation educator, an emergency medicine physician and a resident with BLS, and ACLS certifications. Then, all data were combined, refined, and simplified on a single electronic database. Demographics of the students were then transferred to the same datasheet.

\section{Outcomes}

Each team was evaluated with the ACLS scenario detailed above. ACLS scenario was divided into four cycles to ease the evaluation. Each student in the team was randomly assigned to a specific duty at each cycle and they changed their duties in consecutive cycles. Those duties were: providing chest compressions, giving rescue breaths, defibrillation and drug administration. During the simulation, no intervention, guiding or redirecting was performed by the educators. Simulation educators evaluated the success of each team by assessing specific checkpoints at each cycle. Those checkpoints were determined according to the in-hospital resuscitation algorithm presented in the European Resuscitation Council 2015 Resuscitation guideline. The following key steps of the algorithm were transformed into specific checkpoints: opening the airway and checking breath, checking signs of circulation, activating the resuscitation team, starting and maintaining in-hospital CPR. The success at each checkpoint was combined to determine successful cycles, and those were combined to determine successful scenarios. The primary outcomes were defined as the difference in the proportions of successful cycle and scenario completion rates before and after SBMT.

In the first cycle, the team was supposed to recognize asystole, initiate chest compressions that meet previously defined highquality criteria and successfully manage the airway. The time to chest compression (TCC) and metrics regarding the quality of CPR were the specific checkpoints in this step. In the second cycle, the rhythm was changed to ventricular fibrillation (VF) and the team was supposed to check the rhythm, recognize VF, defibrillate correctly, maintain high-quality chest compressions, and administer the proper dose of epinephrine. In the third cycle, the rhythm was still VF, and the team was supposed to check the rhythm, recognize ongoing VF, defibrillate correctly, administer highquality chest compressions, and proper doses of epinephrine and amiodarone. In the last cycle, the same criteria as the third cycle were sought. At the end of the 4th cycle, if all cycles had been assessed as successful, the return of spontaneous circulation (ROSC) was announced; otherwise scenario was ended with the death of the simulated case.

\section{Definitions and measurements}

The primary endpoint of a successful cycle was determined if airway management was correct, minimum requirements for a high-quality chest compression were met, criteria for correct defibrillation and drug administration were satisfied. If all cycles were assessed as successful, a successful scenario completion was determined. A highquality chest compression (CC) was defined as compression depth and recoil of $50 \mathrm{~mm}$ to $60 \mathrm{~mm}$, and a compression frequency of 100 to $120 \mathrm{bpm}$. Successful airway positioning was determined by the educator according to AHA ACLS criteria. Presence of a successful airway positioning with a ventilation frequency of 10 to 12 was defined as successful airway management. Correct defibrillation was determined by correct positioning and defibrillating with 200 J. Correct drug administration was defined as the proper use of epinephrine, $1 \mathrm{mg}$ IV at 3-5 min intervals, and amiodarone, 300 $\mathrm{mg}$ push and $150 \mathrm{mg}$ repeat dose, at appropriate cycles. Time to chest compression (TCC) was defined as the time in seconds from the start of the simulation to the first compression by the team at the first 
cycle. In other cycles, TCC was defined as the time from the end of defibrillation to the first compression and numbered consecutively. According to the scenario, CC was expected to be initiated at all four cycles. Time to defibrillation (TDEF) was defined as the time in seconds from the moment chest compression was stopped to the moment of defibrillation by the team. According to the scenario, the team was supposed to perform defibrillation at the 2nd, 3rd and 4th cycles.

\section{Statistical analysis}

Continuous variables were reported with medians and interquartile ranges (IQR) since non-normality was observed by Shapiro-Wilks' test. Wilcoxon test was used to compare median values of the variables in before and after groups. Categorical variables were reported as proportions and counts. The differences of proportions, 95\% confidence interval of the difference of proportions and absolute change in the proportions were compared using MonteCarlo simulation for Bayesian inferential statistics, and $p$ values were calculated using z-statistics. In this study, the accepted Type 1 error was $5 \%$. Given the 25 comparisons made in this study we corrected the type 1 error to accept as 0.002 . MedCalc Statistical Software version 17.9.7 was used for statistical analysis (MedCalc Software bvba, Ostend, Belgium; http://www. medcalc.org; 2017).

\section{RESULTS}

In this study, 136 students were evaluated in 34 teams before and after SBMT. Median age was 23 , and $51.5 \%$ were male. The primary outcomes of successfully completed CPR cycles and scenarios were analyzed by Wilcoxon test and presented in table 1 . A statistically significant $60.3 \%(\mathrm{p}=<0.0001)$ increase in the success rate of CPR cycles and $61.8 \%(\mathrm{p}=0.0007)$ increase in successful scenario completion rate was observed after SBMT sessions. The increase in success rates originated primarily from the increase in the rate of adequate chest compression depth $(46.3 \%, \mathrm{p}=<0.0001)$, recoil (46.4\%, $\mathrm{p}=<0.0001)$, and frequency $(52.2 \%$, $\mathrm{p}=<0.0001)$, the increase in the rate of adequate breath frequency $(34.5 \%, \mathrm{p}=<0.0001)$ and accurate positioning of the airway $(30.1 \%, \mathrm{p}=<0.0001)$.

The secondary outcomes of this study were analyzed by Wilcoxon test and presented in table 2 . The change in the median individual data was compared from the 1st cycle to the 4th cycle before and after SBMT. Median time to chest compression (TCC) $(1 \mathrm{sec}$., $\mathrm{p}=<0.0001)$ and median time to defibrillation (TDEF) ( 1 sec., $\mathrm{p}=<0.0001$ ) decreased significantly after SBMT but the difference was prominent at the first attempts (the 1st cycle for TCC and the 2nd cycle for TDEF). The adequacy of chest compressions, compression depth, recoil, and frequency significantly increased after SBMT, $7.0 \mathrm{~mm}(\mathrm{p}=<0.0001), 6.0 \mathrm{~mm}(\mathrm{p}=<0.0001)$ and $8.5 / \mathrm{min}(\mathrm{p}=<0.0001)$, respectively.

\section{DISCUSSION}

Simulation-based medical training (SBMT) is documented to be an effective medical training method. $(12,13)$ Researches have shown that SBMT is a more efficient method when compared with TMT method. $(10,14,15)$ In this study, we compared the efficacy for the combination of SBMT and TMT, with TMT alone in ACLS training in an ACLS scenario.

We examined three critical components of chest compression: compression depth, compression recoil depth, and compression frequency. According to our scenario, only if all three components were performed adequately, the chest compression was accepted as successful since the viability of the heart and other vital organs depend on the CPR quality. Insufficient compression depth, recoil and/or lower rates of compression frequency may cause a decrease in coronary perfusion and aortic peak pressure resulting in poor systemic blood circulation. Nolan et al. and Idris et al., in two different studies, showed that improving chest compression rates is associated with a higher chance for ROSC. $(16,17)$ Our results indicate that medical students improved all three components resulting in a significant increase from $26.5 \%$ to $86.8 \%$ in successful chest compressions after SBMT (tables 1,2). We evaluated airway management in two parts: adequate breath frequency and correct airway positioning. Successful airway management was achieved when both parts were carried out accurately. In 2016 Breuer

Table 1. Comparison of the Success Rate Parameters Before and After SBMT

\begin{tabular}{lllll}
\hline & $\begin{array}{l}\text { Before SBMT } \\
\mathbf{N}(\%)\end{array}$ & $\begin{array}{l}\text { After SBMT } \\
\mathbf{N}(\%)\end{array}$ & $\begin{array}{l}\text { Difference in proportions } \\
(\mathbf{9 5} \% \mathbf{C I})\end{array}$ & $\mathbf{P}^{\star}$ \\
\hline Successful Chest Compressions, $\mathrm{n}(\%)$ & $36(26.5)$ & $118(86.8)$ & $-60.3(-69.2,-49.6)$ & $<0.0001$ \\
\hline Adequate compression depth, $\mathrm{n}(\%)$ & $56(41.2)$ & $119(87.5)$ & $-46.3(-56.3,-36.3)$ & $<0.0001$ \\
\hline Adequate recoil depth, $\mathrm{n}(\%)$ & $55(40.4)$ & $118(86.8)$ & $-46.4(-56.2,-35.3)$ & $<0.0001$ \\
\hline Adequate compression frequency, $\mathrm{n}(\%)$ & $53(39.0)$ & $124(91.2)$ & $-52.2(-61.4,-41.6)$ & $<0.0001$ \\
\hline Successful Airway management, n (\%) & $65(47.8)$ & $124(91.2)$ & $-43.4(-56.7,-29.1)$ & $<0.0001$ \\
\hline Adequate breath frequency, $\mathrm{n}(\%)$ & $79(59.1)$ & $126(92.6)$ & $-34.5(-44.0,-24.3)$ & $<0.0001$ \\
\hline True airway positioning, $\mathrm{n}(\%)$ & $87(64.0)$ & $128(94.1)$ & $-30.1(-39.4,-20.4)$ & $<0.0001$ \\
\hline Correct Defibrillation and Drug application & $129(94.9)$ & $133(97.8)$ & $-2.9(-8.3,2.2)$ & 0.2031 \\
\hline Drug dose & $129(94.9)$ & $133(97.8)$ & $-2.9(-8.3,2.2)$ & 0.2031 \\
\hline Drug administration time & $129(94.9)$ & $133(97.8)$ & $-2.9(-8.3,2.2)$ & 0.2031 \\
\hline Successfully completed CPR cycles, $\mathrm{n}(\%)$ & $36(26.5)$ & $118(86.8)$ & $-60.3(-69.2,-49.6)$ & $<0.0001$ \\
\hline Successfully completed scenarios, $\mathrm{n}(\%), \mathrm{n}=34$ & $8(23.5)$ & $29(85.3)$ & $-61.8(-85.0,-17.7)$ & 0.0007 \\
\hline
\end{tabular}

CI, Confidence Interval; CPR, Cardiopulmonary Resuscitation; SBMT, Simulation Based Medical Training.

${ }^{*} \mathrm{p}<0.002$ is set as significant

All defibrillation attempts were correct for placement and dose for both before and after sessions, therefore omitted from the table. All $\mathrm{n}=136$ except successfully completed scenario rate. 
Table 2. Comparison of the Success Rates of Secondary Parameters Before and After SBMT

\begin{tabular}{|c|c|c|c|c|}
\hline & $\begin{array}{l}\text { Before SBMT } \\
\text { Median (IQR) }\end{array}$ & $\begin{array}{l}\text { After SBMT } \\
\text { Median (IQR) }\end{array}$ & $\begin{array}{l}\text { Median } \\
\text { Difference }\end{array}$ & $\mathbf{P}^{\star}$ \\
\hline TCC (sec), n=136 & $8.0(7.0,11.0)$ & $8.0(6.0,10.0)$ & 1.0 & $<0.0001$ \\
\hline 1st cycle, $\mathrm{n}=34$ & $13.0(10.0,17.0)$ & $10.5(8.0,13.0)$ & 2.0 & $<0.0001$ \\
\hline 2nd cycle, $n=34$ & $8.0(7.0,10.0)$ & $7.0(6.0,9.0)$ & 0.0 & 0.0305 \\
\hline 3 rd cycle, $n=34$ & $7.5(6.0,9.0)$ & $7.0(6.0,8.0)$ & 0.0 & 0.4237 \\
\hline 4 th cycle, $\mathrm{n}=34$ & $8.0(6.0,9.0)$ & $7.0(5.0,8.0)$ & 1.0 & 0.2343 \\
\hline TDEF (sec), n=102 & $8.0(6.0,14.0)$ & $8.0(6.0,13.0)$ & 1.0 & $<0.0001$ \\
\hline 2nd cycle, $n=34$ & $14.0(11.0,17.0)$ & $12.0(10.0,14.0)$ & 2.0 & 0.0002 \\
\hline 3rd cycle, $n=34$ & $7.0(5.0,9.0)$ & $6.5(5.0,9.0)$ & 1.0 & 0.0228 \\
\hline 4 th cycle, $n=34$ & $6.0(5.0,8.0)$ & $6.0(5.0,7.0)$ & 0.0 & 0.3392 \\
\hline Compression depth (mm), $\mathrm{n}=136$ & $45.5(38.0,52.0)$ & $54.5(51.0,57.0)$ & 7.0 & $<0.0001$ \\
\hline Recoil depth (mm), n=136 & $44.0(36.0,51.0)$ & $53.0(50.0,55.0)$ & 6.0 & $<0.0001$ \\
\hline Compression frequency (/min), $\mathrm{n}=136$ & $94.0(88.0,107.5)$ & $108.0(103.0,110.0)$ & 8.5 & $<0.0001$ \\
\hline Breath frequency $(/ \mathrm{bpm}), \mathrm{n}=136$ & $10.0(8.0,11.0)$ & $11.0(10.0,12.0)$ & 1.0 & $<0.0001$ \\
\hline
\end{tabular}

IQR, Interquartile range; SBMT, Simulation-based medical training; TCC, Time to chest compression; TDEF, Time to defibrillation. ${ }^{*} \mathrm{p}<0.002$ is set as significant

et al. reported an $86.7 \%$ insufficient airway positioning and $80 \%$ ineffective ventilation in dental students trained only with TMT and underlined the need for SBMT in ACLS training. (8) We found a significant increase in successful airway management rates after SBMT $(47.8 \%, 91.2 \% \mathrm{p}<0.0001)$ (tables 1,2). There was no significant increase in correct defibrillation and correct drug application rates after SBMT (table 1).

The 2015 guidelines of American Heart Association (AHA) recommends that defibrillation and chest compressions should start immediately and interruptions between chest compressions should be minimized. In this study, we evaluated the Tcc and Tdef in every CPR cycle before and after SBMT. We observed a significant decrease in Tcc and Tdef after SBMT (table 2). In a previous paper from USA, Ko et al. observed Tcc and Tdef in 3rd-year medical students in an ACLS course using high fidelity manikins. (18) They found no significant difference in Tcc and Tdef between TMT group and SBMT. The SBMT group in their study did not previously take TMT unlike our SBMT group and we, as authors, think that this may be the origin of the difference between the results of two studies.

In this study, a significant improvement in CPR quality after SBMT was documented. Successfully completed CPR cycles increased from $26.5 \%$ to $86.8 \%$ and successfully completed scenarios increased from $23.5 \%$ to $85.3 \%$ after SBMT (Table 1) which shows the additional impact of SBMT over TMT.

\section{LIMITATIONS}

All medical students in this study managed the same ACLS scenario before and after SBMT. Students may have improved or worsened their scenario management in their second attempt (after SBMT) which can be judged as a limitation. Instructors gave no feedbacks or debriefings between the scenarios, in order not to contribute to the effect of this limitation on the results. All simulation trainings and scenario evaluations were performed on the same high fidelity manikin since all high fidelity manikins in the simulation center were the same in brand and model. This seems to be a limitation because the students' CPR performing skills may have been affected by the elongated manikin experience. However, depending on our results, the improvement in CPR quality after SBMT is so apparent, that we, as authors, think the influence of these limitations on the results is negligible.

\section{CONCLUSION}

Simulation-based medical training (SBMT) has the potential to advance medical education with the notable developments in simulation technologies. (9) SBMT has proved its effectiveness in recent years. On the other hand, TMT is an efficient medical training method, which has been well-experienced over previous decades. Our results suggest that combining SBMT with TMT improves CPR quality significantly and provides medical students with the fundamental knowledge and required skills that can be used accurately in real life situations. Combining SBMT and TMT appears to be an effective training method in ACLS courses since SBMT has an additional impact on improving CPR quality. 


\section{REFERENCES}

1. Lunenfeld E, Weinreb B, Lavi Y, Amiel GE, Friedman M. Assessment of emergency medicine: a comparison of an experimental objective structured clinical examination with a practical examination. Med Educ 1991;25(1):38-44.

2. Brennan EE, McGraw RC, Brooks SC. Accuracy of instructor assessment of chest compression quality during simulated resuscitation. CJEM 2016;18(4):276-82.

3. Amacher SA, Schumacher C, Legeret C, Tschan F, Semmer NK, Marsch S, et al. Influence of gender on the performance of cardiopulmonary rescue teams: a randomized, prospective simulator study. Crit Care Med2017;45(7):1184-91.

4. Kim JH, Kim WO, Min KT, Yang JY, Nam YT. Learning by computer simulation does not lead to better test performance than textbook study in the diagnosis and treatment of dysrhythmias. J Clin Anesth 2002;14(5):395-400.

5. Perkins GD. Simulation in resuscitation training. Resuscitation 2007;73(2):202-11.

6. Eng AJ, Namba JM, Box KW, Lane JR, Kim DY, Davis DP, et al. High-fidelity simulation training in advanced resuscitation for pharmacy residents. Am J Pharm Educ 2014;78(3):59.

7. DeVita MA, Schaefer J, Lutz J, Wang H, Dongilli T. Improving medical emergency team (MET) performance using a novel curriculum and a computerized human patient simulator. Qual Saf Health Care 2005;14(5):326-31.

8. Breuer G, Knipfer C, Huber T, Huettl S, Shams N, Knipfer K, et al. Competency in managing cardiac arrest: A scenario-based evaluation of dental students. Acta Odontol Scand 2016;74(4):241-9.

9. Wayne DB, Didwania A, Feinglass J, Fudala MJ, Barsuk JH, McGaghie WC. Simulation-based education improves quality of care during cardiac arrest team responses at an academic teaching hospital: a case-control study. Chest 2008;133(1):56-61.

10. Ruesseler M, Weinlich M, Muller MP, Byhahn C, Marzi I, Walcher F. Simulation training improves ability to manage medical emergencies. Emerg Med J 2010;27(10):734-8.

11. Yoo HB, Park JH, Ko JK. An effective method of teaching advanced cardiac life support (ACLS) skills in simulation-based training. Korean J Med Educ 2012;24(1):7-14.

12. Yang TM, Kao Y, Wang CT, Chung MH, Lin HJ, Lin SJ, et al. ACLS training: comparison of physicians and nurses with teamworkbased high-fidelity simulation. Am J Emerg Med 2014;32(9):1132-4.

13. Adams AJ, Wasson EA, Admire JR, Pablo Gomez P, Babayeuski RA, Sako EY, et al. A comparison of teaching modalities and fidelity of simulation levels in teaching resuscitation scenarios. J Surg Educ 2015;72(5):778-85.

14. Wayne DB, Butter J, Siddall VJ, Fudala MJ, Linquist LA, Feinglass J, et al. Simulation-based training of internal medicine residents in advanced cardiac life support protocols: a randomized trial. Teach Learn Med 2005;17(3):210-6.

15. Wayne DB, Butter J, Siddall VJ, Fudala MJ, Wade LD, Feinglass J, et al. Mastery learning of advanced cardiac life support skills by internal medicine residents using simulation technology and deliberate practice. J Gen Intern Med 2006;21(3):251-6.

16. Nolan JP, Perkins GD, Soar J. Chest compression rate: where is the sweet spot? Circulation 2012;125(24):2968-70.

17. Idris AH, Guffey D, Aufderheide TP, Brown S, Morrison LJ, Nichols P, et al. Relationship between chest compression rates and outcomes from cardiac arrest. Circulation 2012;125(24):3004-12.

18. Ko PY, Scott JM, Mihai A, Grant WD. Comparison of a modified longitudinal simulation-based advanced cardiovascular life support to a traditional advanced cardiovascular life support curriculum in third-year medical students. Teach Learn Med 2011;23(4):324-30. 